\title{
Coherent Transport through a Quantum Dot Embedded in an Aharonov-Bohm Ring
}

\author{
Kicheon Kangt \\ Max-Planck-Institut für Physik Komplexer Systeme, Nöthnitzer Str. 38, D-01187 Dresden, Germany
}

(May 11, 2018)

\begin{abstract}
We study the coherent transport in multi-terminal mesoscopic Aharonov-Bohm ring with a quantum dot embedded in an arm. Employing the Friedel sum rule for the effective single-particle levels in the quantum dot, we explain some anomalous features which have been observed in the experiment. We attribute these anomalies to the result of nontrivial quantum interference of the quantum dot with the attached ring. Further, we propose a new feature of conductance oscillations which can be a test for the validity of our model.
\end{abstract}

PACS numbers: 73.20.Dx, 73.23.Hk, 72.15.Rn

Resonant tunneling through a quantum dot is of considerable current interest (see e.g. ref. 11 and references therein). The phase coherence of the resonant tunneling cannot be proved directly in the ordinary conductance measurements through a quantum dot because the conductance measures only the ragnitude of the transmission amplitude. Yacoby et al.2 reported the first experimental demonstration that transmission through a quantum dot has a coherent component, using a two-terminal Aharonov-Bohm (AB) interferometer with a quantum dot embedded in one of its arm. In addition to the observation of the coherence, they have found two other striking features which could not be understood well at first. First, the phase of the AB oscillation changes abruptly by $\pi$ whenever the conductance reaches its maximum. Second, the AB oscillations of successive conductance peaks are in phase. It has been shown that the abrupt phase change at resonance can be understood in terms of the phase rigidity enforced by the condition that the two terminal conductance shauld be an even function of the external magnetic field 35 . The second feature of in phase behavior has not been understood well since neither integrable nor chaotic quantum dots are expected to have generically the same phase between successive resonances. Recently, $\mathrm{Wu}$ et a 6 suggested that the in phase behavior originates from the fact that the resonant tunneling through the whole system can be observed only when the phase shift introduced by the resonant state of the dot coincides with the transmission phase of the reference arm.

In the two-terminal structure, a measurement of the transmission phase of the quantum dot itself is not possible because of the phase rigidity enforced by the microreversibility of the transmission coefficient]. Recently, a modified four-terminal geometry has been adopted to measure the transmission phase of the quantum dot 8 . Because the phase rigidity does not exist in this geometry, they could observe continuous phase shifts of the AB oscillations as a function of the plunger gate voltage on the quantum dot. Within a simplified model that the coherent transmission can be described by the sum of two direct paths, the phase evolution within a resonance could be explained by the Breit-Wigner model for the quan- tum dot. On the other hand, they observed two other striking phenomena which have not been understood by the Breit-Wigner model. First, the AB oscillations of the successive resonances are in phase again as in the twoterminal experiment. Second, there is sharp phase drop, by $\pi$, at some point between successive resonances, which is quite different from the phase change at the peaks in the two-terminal experiment.

In this paper, we address the problem of the coherent transmission through a quantum dot embedded in a twoterminal and in a four-terminal $\mathrm{AB}$ ring. By employing the Green's function method 10 in the tight binding model with the Friedel sum rule for the quantum dot, we obtain some anomalous results which also have been observed in the experiments such as in phase behavior and the inter-resonance phase drop. First, we confirm that the in phase behavior arises because the out of phase resonances in the quantum dot do not appear as a conductance peaks due to the destructive interference, as $\mathrm{Wu}$ et al.6 proposed. Second, it is found that the inter-resonance phase drop accompanies quite anomalous $\mathrm{AB}$ oscillation, which cannot be described by a simple sum of two direct paths. We show that multiple path contributions in the interference are very important in the limit of small transmission probability and closely related to the inter-resonance phase drop. Further, we find an anomalous periodicity in the conductance oscillations, with varying the value of the external magnetic flux. From our result, we conclude that, in general, the transmission through the quantum dot cannot be considered separately from that of the whole system containing the ring. The quantum interferences lead to novel phenomena which cannot be understood in terms of the quantum dot only.

The model we study is a multi-terminal AB ring where a quantum dot is embedded in one of its arm, as shown schematically in Fig.1. We adopt a single channel model of spinless electrons. We use tight binding representation with the hopping integral $t$ of which magnitude is taken to be unity here. This model can be applied for a ring where the ring is so narrow that only a few $1 \mathrm{D}$ channels are included in tranmission. This is exactly the situation of the experiments 2 and 8 . Also we neglect electron 
spin. The spin doesn't seem to play a major role in the experiments because it doesn't show an even-odd parity effect for the occupation number of the quantum dot. The quantum dot is modelled by the barrier energy $E_{B}$ and multi-levels in the site of the dot. An equal spacing is assumed for the effective single particle energies in the quantum dot. The periodicity comes from large charging energy, which implies that the Coulomb interaction effects are being considered through the effective single particle levels. That is, the energy levels in the quantum dot are modelled as $E_{l}=E_{0}+l \Delta$ with $l=0,1,2 \cdots$, where $\Delta \sim e^{2} / C$ with $C$ being the capacitance of the quantum dot. Due to the Friedel sum rule, the increment in the occupation number $\delta n$ and the phase shift $\delta \eta$ of the quantum dot levels are related by

$$
\delta \eta=\pi \delta n .
$$

It should be noted that the sum rule is valid in spite of the electron-electron interactions. In a well confined quantum dot characterized by a large value of $E_{B}$ in our model, the charge in the quantum dot is quantized by the charging energy so that we have $\delta n=1$ between adjacent levels. Thus the adjacent levels have opposite parities with each other, which are denoted by solid lines and dotted lines drawn alternatively in Fig.1(b). This phase shift is taken into account in the hopping matrix elements with the neighboring sites. The magnetic flux $\Phi$ appears in the phase factor $e^{ \pm i \varphi}$ of the hopping integral, where $\varphi=2 \pi \Phi / N_{s} \Phi_{0}$ with $\Phi_{0}=h c / e$ and $N_{s}$ being the elementary flux quantum and the number of lattice sites, respectively. The ring is connected to four reservoirs denoted by $L, R, a, b$ by the coupling constant $t_{\alpha}$ $(\alpha=L, R, a, b)$. The coupling strength is characterized by the parameter

$$
\Gamma_{\alpha}=\pi\left|t_{\alpha}\right|^{2} \rho_{\alpha}\left(\varepsilon_{F}\right),
$$

where $\rho_{\alpha}$ and $\varepsilon_{F}$ denote the density of states and the Fermi energy of the reservoir $\alpha$, respectively.

Owing to the relation between scattering amplitude and the Green function, the transmission probability from the left lead to the right lead, $T_{L R}$, can be related to the Green's function connecting the site 1 and the site $N, G_{1 N}$ :

$$
T_{L R}=4 \Gamma_{L} \Gamma_{R}\left|G_{1 N}\left(\varepsilon_{F}\right)\right|^{2} .
$$

Two-terminal system can be studied by taking $\Gamma_{a}=\Gamma_{b}=$ 0 . The two-terminal conductance is proportional to $T_{L R}$ according to the Landauer formula. In the four-terminal geometry, $T_{L R}$ could be measured with an open circuit collector $\left(I_{R}=0\right)$. The Green function $G_{1 N}$ is calculated by using the standard Green fypction technique in the presence of multi-terminal lead $\$$. 10 .

Fig.2(a) displays the transmission probability and its phase $(\theta)$ of the $\mathrm{AB}$ oscillation as a function of the lowest dot level $E_{0}$. In the experiment, $E_{0}$ can be controlled by the external plunger gate on the quantum dot. As observed in the experiments, periodic conductance oscillation due to the charging energy is shown in this figure. The asymmetry of the peaks is the result of the interference with the upper arm. Surprisingly, the period of the oscillation is not $\Delta$ but $2 \Delta$. This implies that the oscillation period corresponds to adding charge $2 e$ to the quantum dot, not $e$ as in the ordinary Coulomb blockade oscillations. In the two-terminal structure, the conductance should be an even function of the external flux, which allows only abrupt phase change of $\pi$. It should be noted that there are two types of abrupt phase changes. One occurs at resonance and the other does at some point between adjacent resonance peaks. With two types of phase changes in a period, the every peak has the same phase in $\mathrm{AB}$ oscillations as observed in the experiments-2. As a result of the interference with the reference arm, only in phase resonances through the quantum dot appear as conductance peaks, while the out of phase resonances don't give rise to conductance peaks because of destructive quantum interference. This explains why the conductance peaks in the experiment should have the same phase in $\mathrm{AB}$ oscillations.

$\mathrm{AB}$ oscillations are more closely inspected in Fig.2(b). In Fig.2(b), the transmission probability as a function of the flux is displayed for several values of $E_{0}$. One can see that the parity of $\mathrm{AB}$ oscillations is changed twice in a period as mentioned above. The phase change at the conductance meaks is now well understood from the previous studies $\mathrm{B}$. Further, we find that the phase change between the peaks accompanies quite anomalous $\mathrm{AB}$ oscillation. That is, at the point of inter-resonance phase change $(X)$, the transmission amplitude is zero for almost every value of the external flux. It means that coherent transmission is nearly absent at this point. This result is quite similar to the experimental observation of that the inter-resonance phase drop accompanies zero amplitude of the $\mathrm{AB}$ oscillation.

In Fig.3, transmission probability and its phase of $\mathrm{AB}$ oscillation in the four-terminal geometry is displayed as a function of $E_{0}$. While a continuous phase shift has been observed in the experiment $\mathrm{B}$, the phase rigidity still exists in our treatment because net current flow through the other reservoirs $a, b$ is not allowed in this formulation. It has been shown that the phase rigidity is preserved even in the presence of inelastic processes through the other leads if the net current flow is zero through these leads $\mathrm{B}$. In our treatment, the behavior of $\mathrm{AB}$ phase is same as that of two-terminal ring, with reduced transmission probability. The reduction of the probability comes from the fact that the additional reservoir plays a role of inelastic scattering center 11 The $\mathrm{AB}$ oscillation patterns which are not displayed here are same with those of two-terminal interferometer (Fig.2(b)) with reduced magnitude of the oscillations. Looking into the $\mathrm{AB}$ oscillations displayed in Fig.2(b) again, one can find that the magneto-conductance curves are far from sinusoidal of the period $\Phi_{0}$ at low transmission region. This means that multiple path interference is important especially at 
low transmission region (see $C, D, X$ in Fig.2(b)). In contrast, near the resonance, the multiple path contributions are relatively small and the $\Phi_{0}$ period due to direct two paths is dominant (see $A, R, B$ in the Fig.2(b)). This suggests that multiple path contributions should not be neglected in the small transmission limit, and it requires more careful analysis. In analyzing their experimental results, the authors of 8 used a simple model of sum of two direct paths, on the basis of their observation that there is no higher order harmonics in the $\mathrm{AB}$ oscillation with period $\Phi_{0} / n(n>1)$. While it seems valid in describing the phase evolution around the peaks, it is still questionable whether this argument is correct in the limit of small amplitude of AB oscillation. Though our treatment is not complete because of the restriction in the allowed phase values, numerical results indicate at least that multiple path interference cannot be neglected at low transmission limit. Further, multiple path contributions are closely related to the inter-resonance phase drop. At the point of inter-resonance phase drop ( $X$ of Fig.2(b)), effects of multiple path interferences are rather drastic, which lead to quite anomalous $\mathrm{AB}$ oscillation pattern.

In Fig.4, we display $T_{L R}$ as a function of $E_{0}$ for several values of the external flux quantum. Interestingly, the periodicity varies as $2 \Delta \rightarrow \Delta \rightarrow 2 \Delta \rightarrow \Delta$ with increasing the value of the flux. For $\Phi=\Phi_{0} / 2$, the locations of the peaks are shifted by $\Delta$ compared to the zero flux case. This is because the ring acquires AB phase $\pi$ due to the flux, so the in phase resonances and out of phase resonances in the quantum dot are reversed. When the periodicity is $\Delta\left(\Phi=\Phi_{0} / 4,3 \Phi_{0} / 4\right)$, the conductance peaks are no longer in phase, and the positions of the peaks do not coincide with the resonance of the quantum dot. This is also the result of interference. We suggest that the validity of the model presented in this paper can be tested experimentally by investigating the feature in Fig.4.

In conclusion, we have investigated the coherent transmission in two-terminal and in four-terminal mesoscopic Aharonov-Bohm ring with a quantum dot embedded in an arm. Employing the Friedel sum rule for the effective single-particle levels in the quantum dot, we have explained some anomalous features which have been observed in the experiment. We have discussed these anomalous features in relation to the nontrivial quantum interference of the quantum dot with the attached ring. Further, we have proposed a new feature of conductance oscillations which can be a test for the validity of our model.

The author thanks H. Schanz for his critical reading of this manuscript, and P. Fulde for his hospitality during his stay in the MPI-PKS. This work has been supported by the KOSEF and in part by the Visitors Program of the MPI-PKS.
* Electronic address : kckang@mpipks-dresden.mpg.de

${ }^{1}$ For a review, see L. P. Kouwenhoven et al., Mesoscopic Electron Transport, Proceedings of the NATO Advanced Study Institute, edited by L. L. Sohn, L. P. Kouwenhoven and G. Schön (Kluwer 1997).

${ }^{2}$ A. Yacoby, M. Heiblum, D. Mahalu and H. Shtrikman, Phys. Rev. Lett. 74, 4047 (1995).

${ }^{3}$ A. L. Yeyati and M. Büttiker, Phys. Rev. B 52, R14 360 (1995).

${ }^{4}$ G. Hackenbroich and H. A. Weidenmüller, Phys. Rev. Lett. 76, 110 (1996).

${ }^{5}$ A. Yacoby, R. Schuster and M. Heiblum, Phys. Rev. B 53, 9583 (1996)

${ }^{6}$ J. Wu et al., Phys. Rev. Lett. 80, 1952 (1998).

${ }^{7}$ M. Büttiker, Phys. Rev. Lett. 57, 1761 (1986).

${ }^{8}$ R. Schuster, E. Buks, M. Heiblum, D. Mahadu, V. Umansky and H. Shtrikman, Nature 385, 417 (1997).

${ }^{9}$ D. S. Fisher and P. A. Lee, Phys. Rev. B 23, 6851 (1981).

${ }^{10}$ For a review of the Green's function method for coherent transport in mesoscopic systems, see e.g. S. Datta, Electronic Transport in Mesoscopic Systems (Cambridge Univ. Press 1995).

${ }^{11}$ M. Büttiker, IBM J. Res. Dev. 32, 63 (1988).

(a)

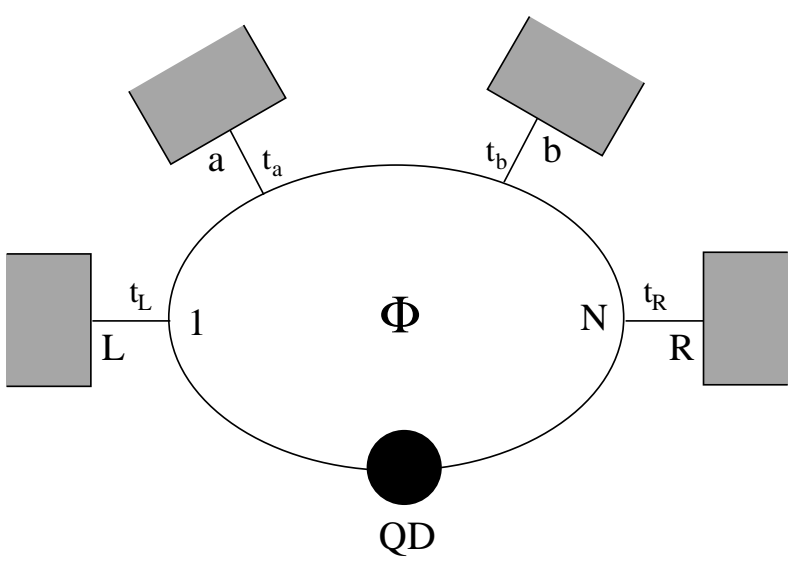

(b)

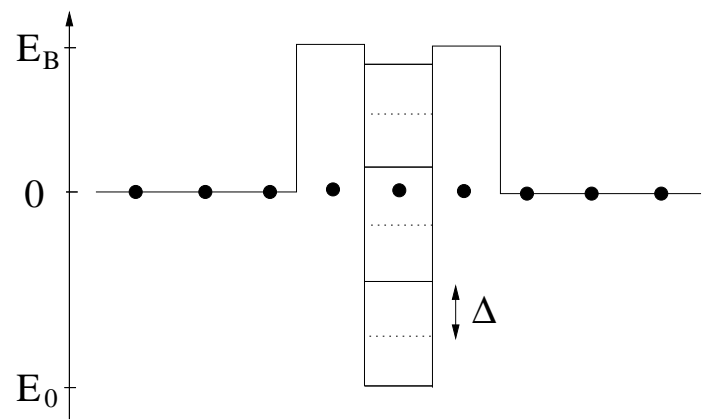

FIG. 1. (a) Schematic diagram of the Aharonov-Bohm ring with a quantum dot coupled to four reservoirs denoted by $L, R, a$ and $b$ with coupling constant $t_{\alpha}(\alpha=L, R, a, b)$. (b) The on-site energies in the tight binding model. The quantum dot is modelled by the barrier energy $E_{B}$ and periodic multi-level energies with its lowest level $E_{0}$ and spacing $\Delta$, respectively. The values of their phase shifts are given by 0 (solid line) and $\pi$ (dotted line) alternatively due to the Friedel sum rule. 

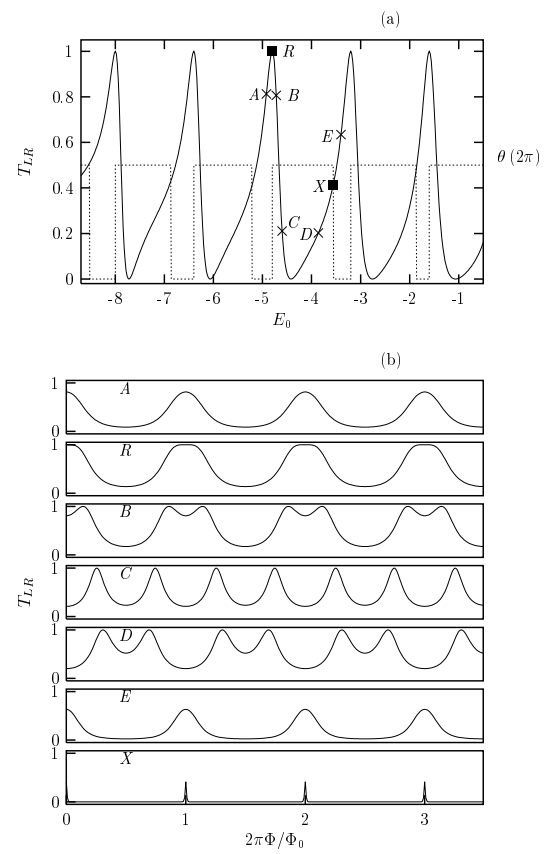

FIG. 2. Transmission probability and phase of AB oscillation as a function of the lowest energy of the dot level $E_{0}$ in the two-terminal ring. The parameters used for the calculations are $\Delta=0.8, \Gamma_{L}=\Gamma_{R}=0.1, \Gamma_{a}=\Gamma_{b}=0$ and $E_{B}=4.0$ in unit of $t . \quad E_{0}$ is also normalized in unit of $t$. 12 levels in the quantum dot are taken into account in the calculation. (a) Transmission probability (solid line) in the absence of the external magnetic flux and its phase of the $\mathrm{AB}$ oscillation (dashed line). (b) AB oscillations of the transmission probability for several values of $E_{0}$ marked as $A, R, B$, $C, D, E$ and $X$ in (a).

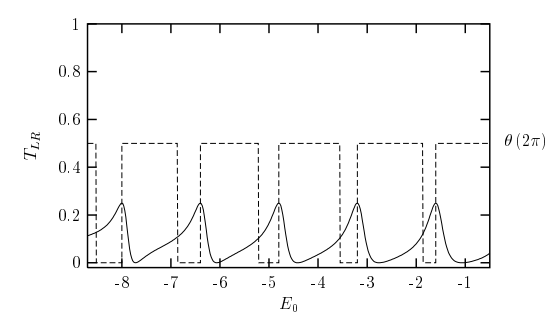

FIG. 3. Transmission probability (solid line) from the left $(L)$ to the right $(R)$ lead with its phase (dashed line) in the four-terminal geometry. The coupling strength is given by $\Gamma_{L}=\Gamma_{R}=\Gamma_{a}=\Gamma_{b}=0.05$, in unit of $t$. Other parameters are same with those in Fig.2.

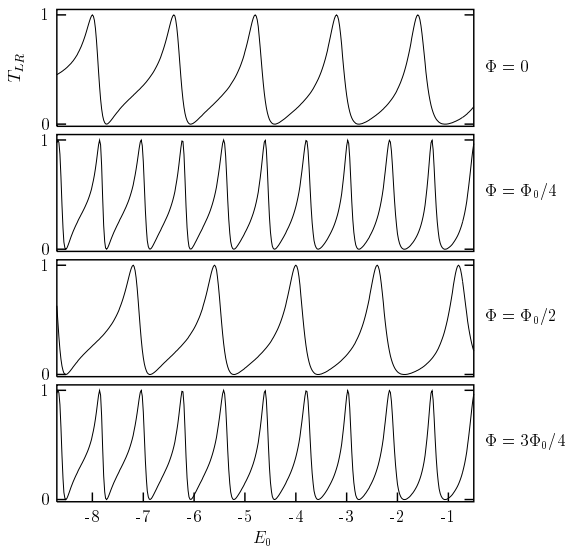

FIG. 4. Transmission probability as a function of $E_{0}$ in the two-terminal geometry for several values of the external flux. Other parameters are same with those in Fig.2. 